\title{
Analysis of Therapeutic Efficacy and Safety of 41 Cases of Chronic Glomerulonephritis Treated by Yishen Jianpi Qufeng Dehumification Decoction and Bailing Capsule
}

\author{
Hongxia Xie ${ }^{1}$, Xin Pang ${ }^{1}$, Xiaoyong Chen ${ }^{1}$, Dongyang $\mathrm{Li}^{1}$, Jianwei Zhang ${ }^{1}$, Yan Zhang ${ }^{2, *}$ \\ ${ }^{1}$ Henan Province Hospital of Traditional Chinese Medicine (The Second Affiliated Hospital of Henan \\ University of Traditional Chinese Medicine), Zhengzhou, Henan, 450003 \\ ${ }^{2}$ The First Affiliated Hospital of Henan University of Traditional Chinese Medicine, Zhengzhou, Henan, \\ 450000
}

Keywords: Yishen Jianpi Qufeng Dehumidification Decoction; Bailing Capsule; Chronic Glomerulonephritis; efficacy and safety

\begin{abstract}
Objective: To investigate the efficacy and safety of Yishen Jianpi Qufeng Dehumification Decoction and Bailing Capsule in the treatment of chronic glomerulonephritis. Methods: Eighty-two cases of chronic glomerulonephritis were enrolled in our hospital from November 2015 to December 2017. The random number table was divided into control group ( $\mathrm{n}=41$ ) and study group $(n=41)$. On the basis of routine intervention, the control group took Bai Ling capsules, and the study group added Yishen Jianpi Qufeng Dehumidification Decoction on the basis of the control group, all of which were treated for 3 months. The clinical efficacy, blood function urea nitrogen (BUN), serum creatinine (Scr), 24-hour urinary protein quantification, and incidence of adverse reactions were measured before and after treatment in both groups. Results: The total effective rate in the study group (92.68\%) was higher than that in the control group (75.61\%) $(\mathrm{P}<0.05)$; the BUN, Scr, and 24-hour urinary protein levels were lower in the study group than before the treatment, and the study group was lower than the control group. There was no significant difference between the incidence of adverse reactions in the study group (12.20\%) and the control group (7.32\%) ( $>0.05)$. Conclusion: The combination of Bailing Capsule and Yishen Jianpi Qufeng Dehumification Decoction in the treatment of chronic glomerulonephritis can effectively improve the renal function of patients and improve the therapeutic effect of the disease. The incidence of adverse reactions is low and safe.
\end{abstract}

\section{Introduction}

Chronic glomerulonephritis is a type of clinically-prone disease. Patients are often accompanied by varying degrees of renal dysfunction. The condition is continuous, slow, and progressive. If the patient is not treated promptly, it will cause end-stage renal failure. Health poses a threat. At present, clinical Western medicine mostly uses angiotensin II receptor antagonists to treat patients with chronic glomerulonephritis, such as telmisartan, valsartan, losartan potassium, etc., can reduce blood uric acid levels, reduce proteinuria, However, the overall efficacy is poor [1]. In recent years, the value of traditional Chinese medicine in the clinical treatment of chronic glomerulonephritis has received widespread attention. Chinese medicine practitioners believe that the disease is a specimen of the same disease, the virtual standard, so the treatment can be blood stasis, basic strength and Qi and other basic Principle [2-3]. Bailing Capsule and Yishen Jianpi Qufeng Dehumidification Decoction are important medicines for the treatment of chronic glomerulonephritis by Chinese medicine. The former can benefit essence gas, supplement lung and kidney, and strengthen immune function. The latter has dampness, extinction, and kidney Spleen and other effects, but no combination of both have been reported in the treatment of chronic glomerulonephritis. Based on this, this study selected 82 patients with chronic glomerulonephritis in our hospital to investigate the efficacy and safety of Yishen Jianpi Qufeng Dehuming Decoction combined with Bailing Capsule. The report is as follows. 


\section{Materials and Methods}

\subsection{General Information.}

In our hospital from November 2015 to December 2017, 82 patients with chronic glomerulonephritis were selected. The random number table was divided into control group $(n=41)$ and study group $(n=41)$. The control group consisted of 24 males and 17 females; aged 23 to 66 years, mean $(44.69 \pm 14.02)$ years; pathological types: 18 cases of mesangial proliferative glomerulonephritis and 15 cases of mesangial capillary glomerulonephritis. Membranous nephropathy is in 8 cases; duration of 0.9 to 6.8 years, an average of (3.89 \pm 1.96$)$ years. The study group included 26 males and 15 females; aged 21 to 69 years, with an average of (45.03 \pm 13.81$)$ years; pathological types: 17 cases with mesangial proliferative glomerulonephritis and 17 cases with mesangial capillary glomerulonephritis. Membranous nephropathy in 7 cases; duration of 1.2 to 7.2 years, mean $(3.96 \pm 2.02)$ years. There was no significant difference in clinical data between the two groups $(\mathrm{P}>0.05)$, such as age, sex, pathological type, and course of disease, and this study was approved by the Ethics Committee of our hospital.

\subsection{Select Criteria.}

Inclusion criteria: (1) Clinically proven to be chronic glomerulonephritis; (2) Knowing the study and signing a consent form. Exclusion criteria: (1) secondary glomerulonephritis and hereditary glomerulonephritis; (2) hematuria; (3) breast-feeding and gestational women; (4) allergies and allergies to study drugs History; (5) Patients with severe renal and hepatic impairment; (6) Malignant tumors.

\subsection{Methods.}

Two groups of patients were given routine interventions after admission, including blood pressure control, low-salt diet, anti-infection, etc., and oral telmisartan (Suzhou Sinochem Pharmaceutical Industry Co., Ltd., Zhuozi H20110157) 80 mg/time, once/ d, on this basis, the two groups take different medication plans.

The control group took Bai Ling capsule (Hangzhou Zhongmei Huadong Pharmaceutical Co., Ltd., Zhunzi Z10910036), orally 1 g/time, 3 times/d.

The study group was treated with Yishen Jianpi Qufeng Dehumidification Decoction on the basis of the control group. The prescriptions of the drug group were: Huaiyi 10 g, Suye $15 \mathrm{~g}$, Atractylodes lancea $10 \mathrm{~g}$, windproof $10 \mathrm{~g}$, blisters $3 \mathrm{~g}$, Chuanxiong $15 \mathrm{~g}$, Atractylodes $15 \mathrm{~g}$, Ligustrum lucidum 15 g, Hawthorn 10 g, Coix seed 30 g, Poria 15 g, Salvia miltiorrhiza 15 g, Loranthaceae 10 g, Eucommia $15 \mathrm{~g}$, Astragalus $30 \mathrm{~g}$, decocted with water, 1 dose/d, Receive $300 \mathrm{ml}$ juice, take it 2 times in the morning and evening. Both groups were treated for 3 months.

\subsection{Efficacy Evaluation Standards.}

24-hour urinary protein quantification is less than $0.2 \mathrm{~g}$, renal function returns to normal levels, and adverse signs and clinical symptoms disappear as clinical control; 24-hour urinary protein quantification Compared with before treatment, it decreased by $\geq 50 \%$, the renal function returned to normal, and the adverse signs and clinical symptoms were relieved. The 24-hour urinary protein quantification was $25 \%$ to $49 \%$ lower than before treatment, and the renal function, signs, and clinical symptoms were decreased. Improvement is effective; failure to the above criteria is invalid; total efficiency $=($ clinical control + markedly + effective $) /$ total number of cases $\times 100 \%$ [4].

\subsection{Observation Indexes.}

(1) After the end of treatment, the clinical efficacy of the two groups was counted. (2) Before and after treatment, the level of renal function was measured. $4 \mathrm{ml}$ of fasting peripheral blood was collected, centrifuged (3000 r/min, $10 \mathrm{~min}$ ), and the supernatant was taken. The 7600-020 ISE automatic biochemistry produced by Hitachi Ltd. was obtained. The analyzer measures blood urea nitrogen (BUN), serum creatinine (Scr), and 24-hour urine protein quantification. (3) statistics the incidence of adverse reactions in the two groups. 


\subsection{Statistical Methods.}

The data were analyzed by SPSS18.0. The measurement data $( \pm \mathrm{s})$ indicated that the $\mathrm{t}$-test, count data $\mathrm{n}(\%)$ indicated, and $\chi 2$ test, $\mathrm{P}<0.05$ indicated that the difference was statistically significant.

\section{Results}

\subsection{Clinical Efficacy.}

The total effective rate in the study group (92.68\%) was higher than that in the control group (75.61\%, $\mathrm{P}<0.05)$. See Table 1.

Table 1 Comparison of clinical efficacy of two groups [n(\%)]

\begin{tabular}{lllllll}
\hline Groups & $\begin{array}{l}\text { Number of } \\
\text { cases }\end{array}$ & $\begin{array}{l}\text { Clinical } \\
\text { control }\end{array}$ & Significantly & Effective & invalid & $\begin{array}{l}\text { Total } \\
\text { efficiency }\end{array}$ \\
\hline $\begin{array}{l}\text { research } \\
\text { group }\end{array}$ & 41 & $11(26.83)$ & $16(39.02)$ & $11(26.83)$ & $3(7.32)$ & $38(92.68)$ \\
$\begin{array}{l}\text { Control } \\
\text { group }\end{array}$ & 41 & $7(17.07)$ & $13(31.71)$ & $11(26.83)$ & $10(24.39)$ & $31(75.61)$ \\
$\chi^{2}$ & - & - & - & - & - & 4.479 \\
$P$ & - & - & - & - & - & 0.034 \\
\hline
\end{tabular}

There was no significant difference in BUN, Scr, $24 \mathrm{~h}$ urinary protein levels between the two groups before treatment ( $\mathrm{P}>0.05)$. After treatment, the urinary protein levels of BUN, Scr, and $24 \mathrm{~h}$ were lower than before treatment. The study group was lower than the control group $(\mathrm{P}<0.05)$. See Table 2.

Table 2 Comparison of renal function indicators in the two groups $( \pm \mathrm{s})$

\begin{tabular}{|c|c|c|c|c|c|}
\hline time & Groups & $\begin{array}{l}\text { Number } \\
\text { of cases }\end{array}$ & $\mathrm{BUN}(\mathrm{mmol} / \mathrm{L})$ & Scr(umol/L) & $\begin{array}{l}24 \text { hUrine protein } \\
\text { quantification(g) }\end{array}$ \\
\hline \multirow{4}{*}{$\begin{array}{l}\text { Before } \\
\text { treatment }\end{array}$} & $\begin{array}{l}\text { research } \\
\text { group }\end{array}$ & 41 & $7.95 \pm 1.19$ & $136.20 \pm 17.33$ & $1.86 \pm 0.33$ \\
\hline & $\begin{array}{l}\text { Control } \\
\text { group }\end{array}$ & 41 & $8.11 \pm 1.25$ & $133.76 \pm 18.05$ & $1.90 \pm 0.35$ \\
\hline & $t$ & - & 0.594 & 0.624 & 0.532 \\
\hline & $P$ & - & 0.554 & 0.534 & 0.596 \\
\hline \multirow{4}{*}{$\begin{array}{l}\text { After } \\
\text { treatment }\end{array}$} & $\begin{array}{l}\text { research } \\
\text { group }\end{array}$ & 41 & $5.03 \pm 0.67$ & $86.12 \pm 12.07$ & $1.09 \pm 0.18$ \\
\hline & $\begin{array}{l}\text { Control } \\
\text { group }\end{array}$ & 41 & $6.58 \pm 0.76$ & $108.41 \pm 13.18$ & $1.41 \pm 0.25$ \\
\hline & $t$ & - & 9.796 & 7.986 & 6.651 \\
\hline & $P$ & - & 0.000 & 0.000 & 0.000 \\
\hline
\end{tabular}

\subsection{Adverse Reactions.}

There was no significant difference between the incidence of adverse reactions in the study group (12.20\%) and the control group (7.32\%) ( $>0.05)$. See Table 3.

Table 3 Comparison of the incidence of adverse reactions in the two groups [n(\%)]

\begin{tabular}{llllll}
\hline Groups & $\begin{array}{l}\text { Number } \\
\text { of cases }\end{array}$ & cough & rash & Vomiting & Total incidence \\
\hline $\begin{array}{l}\text { research } \\
\text { group }\end{array}$ & 41 & $2(4.88)$ & $1(2.44)$ & $2(4.88)$ & $5(12.20)$ \\
Control & 41 & $1(2.44)$ & $0(0.00)$ & $2(4.88)$ & $3(7.32)$ \\
group & - & - & - & - & 0.554 \\
$\chi^{2}$ & - & - & - & - & 0.457 \\
$P$ & - & - & - \\
\hline
\end{tabular}




\section{Discussion}

The incidence of chronic glomerulonephritis is high. Western medicine doctors take benazepril and other drugs to treat patients, which can effectively relieve the clinical symptoms of patients. However, there is a certain gap between the overall curative effect and clinical expectations, and dizziness and itching are prone to occur after treatment. [5].

With the development of Chinese medicine research, the treatment of chronic glomerulonephritis focuses on the treatment of traditional Chinese medicine. Bailing capsules are commonly used. They are extracted from Cordyceps sinensis strains, and are processed by low-temperature fermentation. The chemical composition is similar to that of natural Cordyceps, including Trace elements, vitamins, sugars, ergotol, amino acids, etc. Related studies have shown that Bailing capsules can promote epithelial cells in renal tubular epithelial cells, enhance immune activity, improve renal tubular function, promote epithelial cell repair, inhibition of renal interstitial fibrosis, renal tubular atrophy, delay the deterioration of renal function [6 ]. At the same time, adenosine in Bailing capsules can increase superoxide dismutase, reduce lipid peroxide content, scavenge oxygen free radicals, reduce the degree of kidney damage, improve renal blood circulation, inhibit platelet aggregation, and repair kidney cells [7].

Yishen Jianpi Qufeng Dehumidification Decoction is also commonly used in the treatment of chronic glomerulonephritis. The prescription is treated with astragalus as a king drug, which is beneficial to water detumescence and spleen-invigorating kidney function; Atractylodes can strengthen the spleen function and used together with sputum. Can also have a protective effect on Stomach; Du Chung strong gluten Zhuang bone, conditioning Chong Ren, replenishing liver and kidney, "Shen Nong Ben Cao Jing" contains: "Eu... Indications waist and knee pain, make up, Yi Jing Jing, strong bones and muscles; "Hawthorn is a strong, astringent drug, with astringent fine solid off, tonic of the liver and kidney; Ligustrum lucidum can make up liver and kidney yin; Atractylodes lanceolata, Coix seed, Morus parasitica are dehumidification drugs, which Sansha parasitic can be strong bones and bones, Yigan Kidney, Yiyiren can spleen to stop bleeding, water diuresis, Atractylodes dripping cold, spleen dampness. In addition, Yishen Jianpi Qufeng Dehumidification Decoction, Chuanxiong for the gas in the blood drug, can relieve wind pain, good governance rheumatic pain rheumatism; leech protein and hirudin can pass through, scattered stasis, promoting blood circulation; Stop phlegm, evacuation of wind and heat; Su Ye beneficial spleen and stomach, through the heart through the effect; wind and wind can win over pain, hurricane solution table. Song Huanping[8] research pointed out that Yishen Jianpi Qufeng Dehumidification Decoction is rigorous. All medicines can be combined with dehumidification, qi and spleen, which can effectively reduce the clinical symptoms of patients with chronic glomerulonephritis. The results of this study showed that after the end of treatment, the level of renal function in the study group was lower than that in the control group, and the clinical efficacy was better than that in the control group $(\mathrm{P}<0.05)$, indicating that combination of Bai Ling Capsule and Yishen Jianpi Qufeng Dehumidification Decoction on chronic glomeruli The treatment of nephritis can effectively improve the patient's renal function and improve the treatment effect of the disease. According to the results of the study, there was no significant difference in the incidence of adverse reactions between the study group and the control group $(\mathrm{P}>0.05)$, suggesting that the combination therapy of Bailing capsule and Yishen Jianpi Qufeng Dehumola Decoction can not only be used in chronic glomerulonephritis. Achieve good results without increasing the risk of adverse reactions and high safety.

In summary, the use of Bailing capsule combined with Yishen Jianpi Qufeng Dehumction Decoction in the treatment of chronic glomerulonephritis can effectively improve the patient's renal function, improve the efficacy of disease treatment, and the incidence of adverse reactions is low, with safety.

\section{References}

[1] Liu Fang, Zeng Huifen, Ran, et al. Effect of Bailing capsules combined with alprostadil on 
urinary protein levels and renal function in patients with primary chronic glomerulonephritis[J]. Clinical Journal of Integrated Traditional Chinese and Western Medicine, 2017, 17(6):127-128.

[2] Jiang Junyan, Li Xinling, Fan Zefeng, et al. Meta-analysis of the clinical efficacy of Bailing capsule combined with western medicine in the treatment of chronic glomerulonephritis[J].Journal of Hunan University of Traditional Chinese Medicine,2017,37(12):1363-1368.

[3] Zhou Yingyong, Zhao Dian. Effect of telmisartan combined with Bailing capsule on chronic glomerulonephritis and its effect on renal function [J]. Evaluation and Analysis of Drug Use in Chinese Hospitals, 2017, 17(9): 1211-1212.

[4] Zheng Zheng. Guiding Principles for Clinical Research of New Chinese Drugs (Trial)[M]. Beijing: China Medical Science and Technology Press, 2002:156-163.

[5] Zhu Hong, Jin Lie, Mao Mingfeng. Effect of Bailing capsule combined with benazepril on the efficacy and renal function of patients with chronic glomerulonephritis [J]. China Modern Doctor, 2017, 55(15): 91-94.

[6] Liu Dajun, Liu Yushi. Observation of the short-term curative effect of telmisartan combined with Bailing capsule in the treatment of chronic glomerulonephritis [J]. Chinese Journal of Practical Internal Medicine, 2015, 35(6):528-530.

[7] Zhang Wenyu, Li Jinping, Chang Wenxiu.Effects of Shenfukang capsule combined with bailing capsule on chronic glomerulonephritis [J]. Modern Medicine \& Clinical Medicine, 2016, 31(6): 823-825.

[8] Song Huanping. Clinical efficacy of Yishen Jianpi Qufeng Dehuming Decoction combined with benazepril in the treatment of chronic glomerulonephritis [J].Journal of Clinical Rational Drug Application, 2017, 10(6):117-119. 